\title{
Advanced glycation end products promote osteoporosis by inducing ferroptosis in osteoblasts
}

\author{
WEIWEI GE ${ }^{1 *}$, JIAN JIE ${ }^{2 *}$, JIE YAO $^{3}$, WEI LI ${ }^{1}$, YAHUI CHENG ${ }^{1}$ and WENJUAN LU ${ }^{3}$ \\ Departments of ${ }^{1}$ Radiology and ${ }^{2}$ Orthopedics, Pukou Branch of Jiangsu People's Hospital \\ (Nanjing Pukou District Central Hospital); ${ }^{3}$ Department of Radiology, Nanjing Central Hospital \\ (Nanjing Municipal Government Hospital), Nanjing, Jiangsu 210018, P.R. China
}

Received November 1, 2021; Accepted December 3, 2021

DOI: $10.3892 / \mathrm{mmr} .2022 .12656$

\begin{abstract}
Advanced glycation end products (AGEs) have been widely reported to play an important role in osteoporosis (OP), particularly in diabetes-related OP. The aim of the present study was to investigate the effect of AGEs on osteoblast function and the underlying mechanisms. The level of bone mineral density (BMD), serum AGEs and fasting blood glucose (FBG) was measured in patients with OP and healthy individuals, and the correlation between AGE levels and BMD or FBG was then analyzed. For the in vitro experiments, the hFOB1.19 osteoblast cell line was cultured in medium containing AGEs and serum from healthy individuals or patients with OP, and with or without type-2 diabetes mellitus (T2DM). Cell proliferation, differentiation, mineralization, apoptosis and ferroptosis were evaluated using Cell Counting Kit- 8 and alkaline phosphatase (ALP) assays, Alizarin red and TUNEL staining, iron indicator, lipid peroxidation tests and western blot analysis, respectively. In a separate set of experiments, the ferroptosis inhibitor, deferoxamine (DFO), was also added to the culture medium of cells treated with AGEs and serum from patients with OP and T2DM. The results demonstrated that patients with OP had a higher level of serum AGEs and FBG compared with that in healthy individuals. The level of serum AGEs in patients with OP was negatively correlated with BMD, but was positively correlated with FBG. In addition, AGEs and serum from patients with OP markedly inhibited hFOB1.19 cell proliferation, ALP production and mineralized nodule formation. Apoptosis and ferroptosis were significantly promoted by AGEs and serum from patients with OP. Moreover, serum from OP patients with T2DM caused stronger effect than that
\end{abstract}

Correspondence to: Dr Wenjuan Lu, Department of Radiology, Nanjing Central Hospital (Nanjing Municipal Government Hospital), 116 Chengxian Street, Xuanwu, Nanjing, Jiangsu 210018, P.R. China E-mail: luwenjuan300@163.com

*Contributed equally

Key words: advanced glycation end products, ferroptosis, osteoblast, osteoporosis, type-2 diabetes mellitus from OP patients with normal FBG. However, DFO reversed the effects induced by AGEs and serum from patients with OP and T2DM on hFOB1.19 cells. Collectively, AGEs could disrupt the functions of osteoblasts by inducing cell ferroptosis, thus contributing to OP.

\section{Introduction}

Osteoporosis (OP) is a systemic skeletal disease characterized by decreased bone mass and microarchitectural deterioration in the bone tissue, which predisposes affected individuals to increased bone fragility and fracture risk (1). The diagnosis of OP is based on bone mineral density (BMD) measurement using dual-energy X-ray absorptiometry (DXA). According to the World Health Organization, a BMD T-score of $<-2.5$, as measured by DXA, is defined as OP (2). Approximately 50\% of women experience at least one bone fracture following menopause worldwide in 2020; OP has become a significant public health problem and received considerable attention worldwide (3).

Type-2 diabetes mellitus (T2DM) has been hypothesized to cause decreased BMD (4). A large prospective study of post-menopausal women recruited into the Study of Osteoporotic Fractures confirmed that female patients with T2DM experienced higher fracture rates compared with that in female patients without T2DM (5). In addition, BMD decreased as diabetes progressed, suggesting that hyperglycemia is an important risk factor for OP (6).

Advanced glycation end products (AGEs) are a group of modified proteins and/or lipids with damaging potential, that are formed under hyperglycemic conditions (7). AGEs have been largely reported to be involved in the pathogenesis of T2DM and diabetic complications (7). In addition, increasing evidence indicates that AGEs play a role in aggravating bone fragility, as observed during the progression of classic diabetic complications $(8,9)$. AGEs have been found to significantly inhibit osteoblast proliferation, differentiation and mineralization and induce osteoblast apoptosis $(10,11)$. AGEs can affect osteoblast proliferation and function by modulating autophagy via the receptor of AGEs (RAGE) pathway, indicating that autophagy is involved in these processes (12). These observations suggest that the AGE/RAGE axis may play an important role in bone formation. 
A recent study have revealed that another form of regulated cell death, ferroptosis, may be involved in the pathogenesis of OP (13). Ferroptosis (iron-dependent cell death) differs from apoptosis, autophagy, necrosis, necroptosis and pyroptosis, and is characterized by the accumulation of intracellular iron and lipid reactive oxygen species (14). The increase in osteoblast ferroptosis plays an important role in hormone-induced OP (15), and iron overload plays an important role in general OP and osteoblast function (16). In a rat model of T2DM-related OP (OP-T2DM), ferroptosis was observed in the bone tissue (17). However, none of the aforementioned studies directly studied the level and changes of ferroptosis in osteoblasts.

Therefore, the aim of the present study was to investigate the role of ferroptosis in AGE-regulated dysfunction of human osteoblasts. Ex vivo experiments using clinical samples from patients with OP and healthy individuals were performed. In addition, in vitro experiments using the hFOB1.19 osteoblast cell line, which was cultured in media containing AGEs and serum from healthy individuals or patients with OP and a normal fasting blood glucose (FBG) level and patients with OP and T2DM to observe cell differentiation, mineralization, apoptosis and ferroptosis, were also performed.

\section{Materials and methods}

Sample collection. The present study was approved by the Ethics Committee of the Nanjing Pukou District Central Hospital (Nanjing, China). Written informed consent was obtained from all participants. A total of 10 healthy individuals and 10 patients with OP (among which 4 were diagnosed with T2DM) were enrolled into the present study. Participants with a femoral neck and/or lumbar spine T score $\leq-2.5$ were considered to have $\mathrm{OP}$, while a $\mathrm{T}$ score of $>-1$ was considered normal. Patients with malignancy, coronary heart disease or other severe diseases in the previous 5 years were excluded. Images of lumbar vertebra and femur, along with the BMD from healthy individuals and patients with OP, were obtained using DXA absorptiometry (Hologic, Inc.). Blood samples from the enrolled participants were centrifuged at $1,000 \mathrm{x}$ for $10 \mathrm{~min}$ at $4^{\circ} \mathrm{C}$, and the serum was stored at $-80^{\circ} \mathrm{C}$.

Measurement of AGEs and FBG. All the participants fasted from 10:00 pm the previous night, and $10 \mathrm{ml}$ venous blood was collected at 29:00 am the next morning. The blood samples were centrifuged at $1,000 \mathrm{x}$ for $10 \mathrm{~min}$ at $4^{\circ} \mathrm{C}$, and the serum was used to assess AGEs and FBG or stored at $-80^{\circ} \mathrm{C}$ for subsequent experiments. The concentration of AGEs and FBG in the serum samples was measured using an AGE assay kit (cat no ab238539; Abcam) and a blood glucose kit (Glucose Oxidase Peroxidase Assay; cat no WD-0108 Shanghai Rongbai Biotechnology Co., Ltd.), following the manufacturer's instructions.

Preparation of AGEs. AGEs were prepared as previously described (18). Briefly, human serum albumin $(15 \mathrm{mg} / \mathrm{ml}$; Sigma-Aldrich; Merck KGaA) was incubated with $1 \mathrm{mM}$ 3-deoxygluconaldeone in distilled water at $37^{\circ} \mathrm{C}$ for 14 days.
Cell culture and grouping. The human hFOB1.19 osteoblast cell line (American Type Culture Collection; ATCC) was cultured in medium containing 1:1 mixture of Ham's F12 medium and DMEM (both from Thermo Fisher Scientific, Inc.) with $2.5 \mathrm{mM}$ L-glutamine (ATCC), supplemented with 10\% FBS (Gibco; Thermo Fisher Scientific, Inc.), $100 \mathrm{U} / \mathrm{ml}$ penicillin, $100 \mathrm{U} / \mathrm{ml}$ streptomycin (Merck KGaA) and $0.3 \mathrm{mg} / \mathrm{ml} \mathrm{G} 418$ (Merck KGaA). The cells were cultured at $37^{\circ} \mathrm{C}$ in a humidified incubator with $5 \% \mathrm{CO}_{2}$.

The hFOB1.19 cells were divided into different groups: Blank (cultured in normal medium), AGEs (cultured in medium containing $50 \mathrm{pg} / \mathrm{ml}$ AGEs), control (cultured in medium containing $10 \%$ serum from healthy individuals), OP-normal (cultured in medium containing 10\% serum from patients with OP and a normal FBG level), OP-T2DM (cultured in medium containing $10 \%$ serum from patients with OP and T2DM), AGEs + deferoxamine (DFO; cultured in medium containing $50 \mathrm{pg} / \mathrm{ml}$ AGE and $80 \mu \mathrm{M}$ DFO), OP-T2DM + DFO (cultured in medium containing $10 \%$ serum from patients with OP and T2DM, and $80 \mu \mathrm{M}$ DFO).

Cell counting kit (CCK)- 8 assay. The hFOB1.19 cell line, in the logarithmic growth phase, were seeded into 96-well plates, at a density of $3 \times 10^{3}$ cells/well, with five replicate wells in each group. Following culture for $24 \mathrm{~h}$ in normal medium, the medium was replaced with new medium according to the aforementioned groups. Following culture for 0, 24, 48 and $72 \mathrm{~h}, 10 \mu \mathrm{l} \mathrm{CCK}-8$ solution (Beyotime Institute of Biotechnology) was added to each well and the samples were incubated at $37^{\circ} \mathrm{C}$ in the dark for $2 \mathrm{~h}$. The absorbance value of each well was measured at $450 \mathrm{~nm}$ using a microplate reader (Bio-Rad Laboratories, Inc.).

Alkaline phosphatase (ALP). The ALP level in the hFOB1.19 cell lysate from each group was measured using a commercial ALP Assay kit (cat no ab83369; Abcam), following the manufacturer's instructions. Briefly, following culture in different media for $48 \mathrm{~h}$, the cell supernatant was removed and the production of p-nitrophenyl phosphate was determined by measuring the absorbance at $405 \mathrm{~nm}$ using a microplate reader (Bio-Rad Laboratories, Inc.).

Alizarin red staining. The bone mineralization of the hFOB1.19 cells in each group was detected using Alizarin red staining (Merck KGaA), following the manufacturer's instructions. Briefly, the cells were stained with 2\% Alizarin red $(\mathrm{pH} \mathrm{4.2)} \mathrm{for} 10 \mathrm{~min}$ at room temperature, then washed with distilled water. The mineralized nodules were examined using phase-contrast microscopy after culturing for 21 days.

TUNEL staining. Cell apoptosis was evaluated using a TUNEL assay (TUNEL Assay kit-FITC; cat. no. ab66108; Abcam), according to the manufacturer's instructions. Briefly, following culture in different media for $48 \mathrm{~h}$, the cells were trypsinized, washed with PBS and fixed in $1 \%$ paraformaldehyde at $4^{\circ} \mathrm{C}$ for $2 \mathrm{~h}$. The cells were then incubated with DNA labeling solution for $60 \mathrm{~min}$ at $37^{\circ} \mathrm{C}$, followed by the addition of PI/RNase A solution for $30 \mathrm{~min}$. The images of the TUNEL-labeled cells from 3 random 
fields were captured using a fluorescent microscope (magnification, x200; Evos FL Cell Imaging System; Thermo Fisher Scientific, Inc.).

Determination of iron level. The ferrous iron level in the cells was determined using a commercially available indicator (cat. no. P14312; Thermo Fisher Scientific, Inc.), as previously described (19). Briefly, following treatment, the cells were resuspended in normal medium, then Phen Green ${ }^{\mathrm{TM}}$ SK fluorescent iron chelator was added for $15 \mathrm{~min}$. The fluorescence represented the release of free iron and was observed at the excitation/emission values of 490/520 nm using a fluorescence microscope (magnification x200; Evos FL Cell Imaging System; Thermo Fisher Scientific, Inc.).

Malondialdehyde (MDA) and oxidized low density lipoprotein (oxLDL) assessment. The relative MDA and oxLDL levels in the hFOB1.19 cells were detected using a Lipid Peroxidation (cat. no. ab118970) and oxLDL (cat. no. ab242302) (both from Abcam) assay kits, respectively, according to the manufacturer's instructions.

Glutathione (GSH)/oxidized GSH (GSSG) assay. The relative GSH concentration in the cells was assessed using a GSH/GSSG Ratio Detection Assay kit (Fluorometric-Green; cat. no. ab138881; Abcam), according to the manufacturer's instructions. Briefly, the whole cell lysates were diluted at 1:80 for GSH analysis, and a series dilution of GSH and GSSG stock standards were prepared as standards. A one-step fluorimetric reaction was performed, with the assay buffer and probes, for $30 \mathrm{~min}$ at room temperature in the dark. Fluorescence intensity was monitored at the excitation/emission values of $490 / 520 \mathrm{~nm}$. GSH was calculated from the standard curve. GSSG was determined using the following equation: GSSG=(total GSH-GSH)/2.

Western blot analysis. Following treatment, the cells were lysed in cold RIPA buffer (Beyotime Institute of Biotechnology). The protein lysates were then centrifuged at $12,000 \mathrm{x} \mathrm{g}$ for $10 \mathrm{~min}$ at $4^{\circ} \mathrm{C}$ and the supernatant was collected. Protein concentration was determined using a BCA assay kit (Beyotime Institute of Biotechnology). A total of $10 \mu \mathrm{g}$ total protein was separated using $10-15 \%$ SDS-PAGE, then transferred onto PVDF membranes. The membranes were then blocked for $1 \mathrm{~h}$ at room temperature with 5\% skimmed milk and subsequently incubated with the primary antibodies overnight at $4^{\circ} \mathrm{C}$. Finally, the membranes were incubated with a HRP-conjugated secondary antibody (goat anti-rabbit IgG; cat no. ab6721; Abcam; 1:10,000) for $2 \mathrm{~h}$ at room temperature. The bands were detected using the ECL Advance reagent (Cytiva) and quantified using ImageJ software (version 1.8.0; National Institutes of Health). The following primary antibodies were used: GSH peroxidase (GPX4; ab125066; Abcam; 1:5,000), solute carrier family 7 member 11 (SLC7A11; ab175186; Abcam; 1:5,000), nuclear receptor coactivator 4 (NCOA4; ab86707; Abcam; 1:3,000), acyl-CoA synthetase long-chain family member 4 (ACSL4; ab15282; Abcam; 1:10,000), transferrin receptor (TFR1), divalent metal transporter 1 (DMT1; ab214039; Abcam; 1:1,000) and GAPDH (ab181602; Abcam; 1:1,000).
Statistical analysis. All the data are from 3 experiments and are presented as the mean $\pm \mathrm{SD}$. Comparisons between two groups were analyzed using an unpaired Student's t-test. Comparisons among multiple groups were evaluated using one-way ANOVA followed by Tukey's post hoc test. $\mathrm{P}<0.05$ was considered to indicate a statistically significant difference. All statistical analysis was performed using SPSS software (version 11.5; SPSS, Inc.).

\section{Results}

Level of serum AGEs and FBG is increased in patients with $O P$. Fig. 1A shows the images of lumbar vertebra and femur in healthy individuals and patients with OP, which were collected during DXA absorptiometry. Compared with that in the healthy individuals, patients with OP exhibited a decreased BMD in both the lumbar vertebra and femur. Fig. 1B reveals the significantly lower BMD in patients with OP compared with that in the healthy individuals. Next, the level of AGE and FBG in the serum of the participants were measured. Patients with OP exhibited a significantly higher level of AGEs (Fig. 1C) and FBG (Fig. 1D). The correlation between BMD and AGEs, and between FBG and AGEs in patients with OP was analyzed and the results showed that in patients with OP, the higher the AGE level, the lower the BMD level, but the higher the FBG level (Fig. 1E and F, respectively). These results suggested that an increase in the concentration of AGEs and FBG may be associated with the decrease in BMD, and even contribute to OP.

AGEs and serum from patients with OP inhibit the proliferation, differentiation and mineralization of hFOB1.19 osteoblasts. To validate the aforementioned hypothesis in vitro, the hFOB1.19 osteoblast cell line was cultured in normal medium (blank), medium containing AGEs (AGEs), or media containing serum from healthy individuals (control), OP patients with a normal FBG level (OP-normal) and OP patients with T2DM (OP-T2DM). Cell proliferation, differentiation and mineralization were then assessed. The cells that were treated with AGE, and serum from patients with OP and T2DM exhibited significantly reduced cell proliferation ability $24 \mathrm{~h}$ post-treatment, compared with that in cells in the blank and control groups, respectively (Fig. 2A). Compared with that in serum from the healthy individuals, serum from patients with OP and a normal FBG level had significantly lower cell proliferation $48 \mathrm{~h}$ post-treatment $(\mathrm{P}<0.05)$. In addition, the serum from patients with OP and T2DM resulted in a relatively higher decrease in cell proliferation compared with that in serum from patients with OP and a normal FBG level (Fig. 2A). A similar result was found for ALP levels in the hFOB1.19 cells (Fig. 2B), in which ALP level was decreased by AGEs and serum from patients with OP (OP-Normal and OP-T2DM). Alizarin red staining was performed to determine the mineralization of osteoblasts. Compared with that in cells in the blank and control groups, cells in the AGE, OP-Normal and OP-T2DM groups exhibited a decrease in the number of mineralized nodules (Fig. 2C). These results suggested that AGEs and serum from patients with OP could inhibit the proliferation, differentiation and mineralization of hFOB1.19 osteoblasts. In addition, the effect of serum from patients with OP and T2DM was stronger compared with that of serum from patients with OP and a normal FBG level. 
A
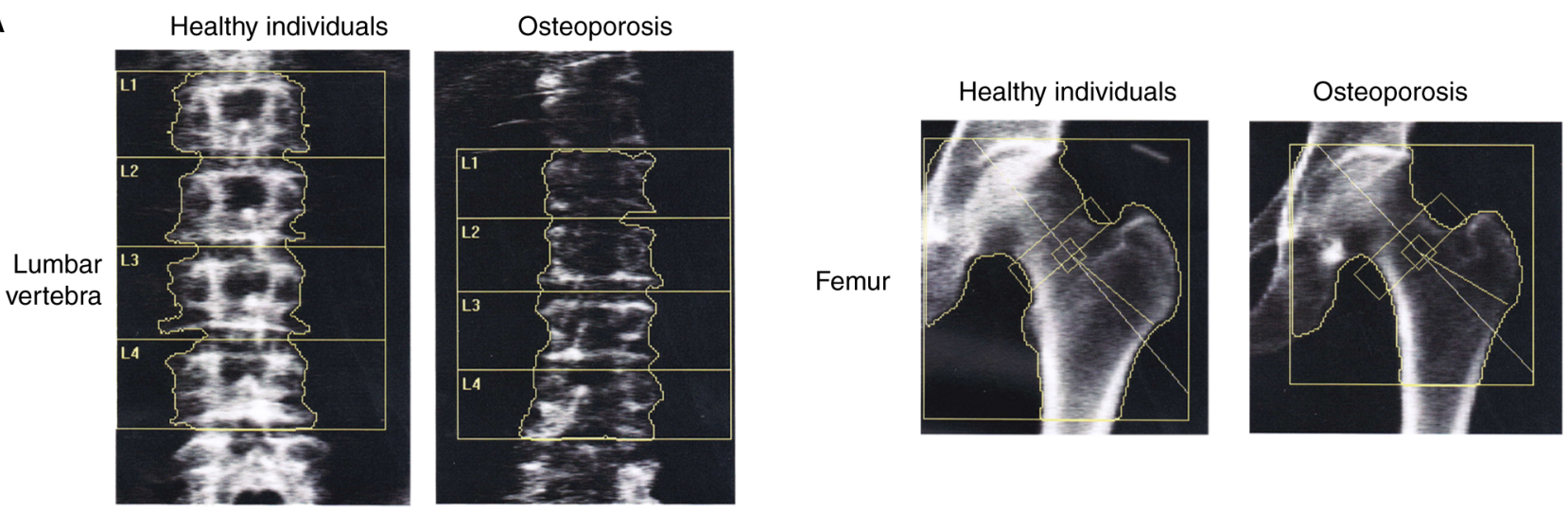

B
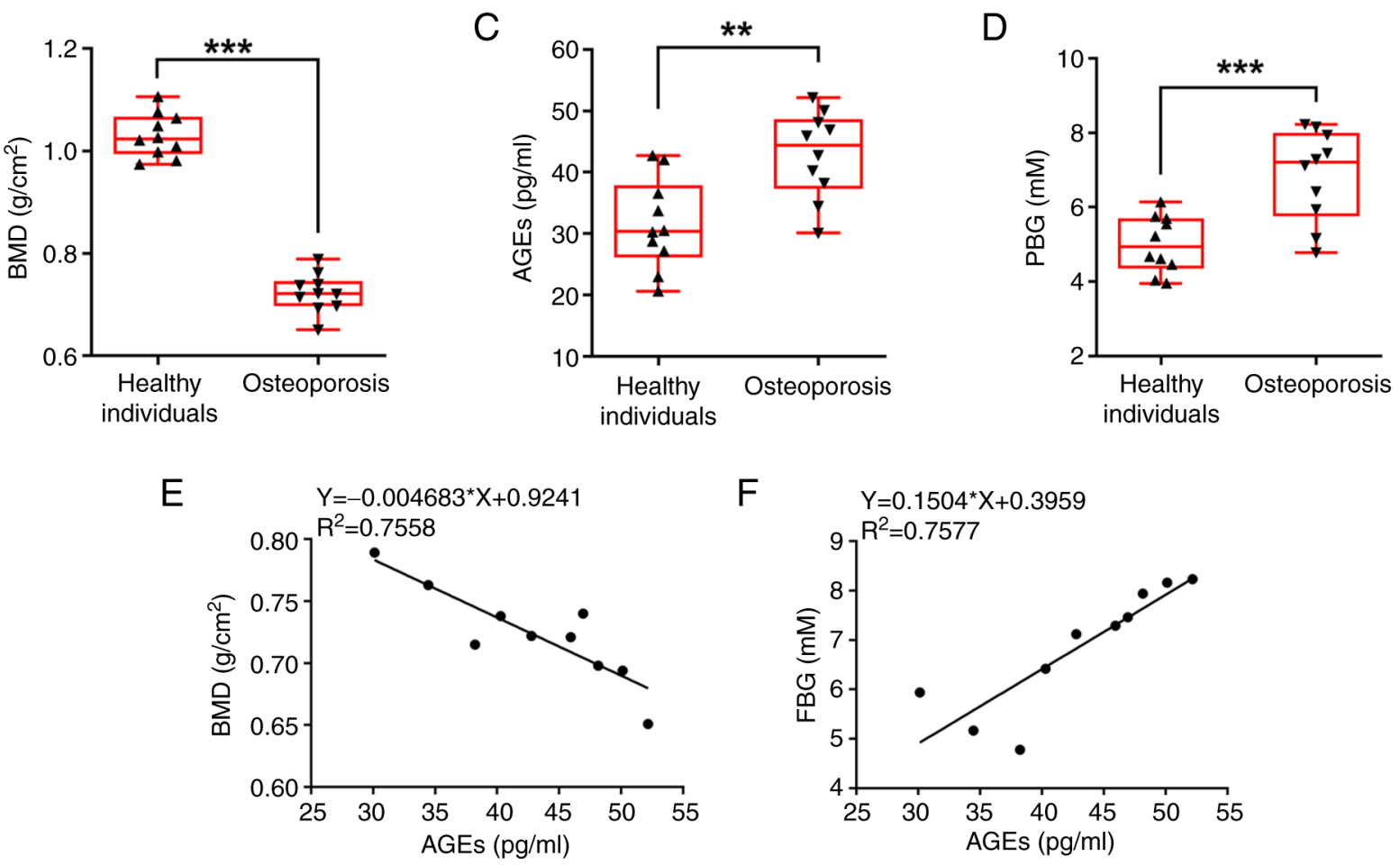

Figure 1. Patients with OP exhibit an increased level of serum AGEs and FBG compared with that in healthy individuals. (A) Images of lumbar vertebra and femur in healthy individuals, patients with OP, and patients with osteopenia were collected using DXA absorptiometry. (B) The BMD of healthy individuals and patients with OS was measured using DXA absorptiometry. (C) AGE and (D) FBG serum levels in healthy individuals and patients with OP. Correlation between (E) BMD and AGEs, and (F) FBG and AGEs, in patients with OP. ${ }^{* *} \mathrm{P}<0.01$ and ${ }^{* * *} \mathrm{P}<0.001$. BMD, bone mineral density; AGEs, advanced glycation end products; FBG, fasting blood glucose; DXA, dual-energy X-ray absorptiometry; OP, osteoporosis.

AGEs and serum from patients with $O P$ induce ferroptosis in hFOB1.19 osteoblasts. Subsequently, changes in cell ferroptosis were investigated. TUNEL staining was used to observe cell apoptosis. As shown in Fig. 3A, the AGEs group had a higher percentage of apoptotic cells compared with that in the blank group. The OP-Normal and OP-T2DM groups also exhibited increased apoptosis compared with the control group. Next, cellular free iron levels were analyzed (Fig. 3B) and the results showed that AGEs and serum from patients with OP significantly increased the cellular free iron level of hFOB1.19 osteoblasts. The concentrations of MDA and oxLDL was also significantly increased by AGEs and serum from patients with OP (OP-Normal and OP-T2DM), compared with those in the blank and control groups, respectively (Fig. 3C and D). However, the opposite effect was caused by AGEs and serum from OP patients (OP-Normal and OP-T2DM) on GSH/GSSG level (Fig. 3E). Following the detection of the expression level of ferroptosis-associated proteins, it was found that AGEs and serum from patients with OP resulted in a decrease in the protein expression level of GPX4 and SLC7A11, but an increase in the protein expression level of NCOA4, ACSL4, TFR1 and DMT1 (Fig. 3F and G). The results revealed that AGEs and serum from patients with OP significantly increased ferroptosis in hFOB1.19 osteoblasts, and that the effect of the serum from patients with $\mathrm{OP}$ and T2DM was stronger compared with that from patients with OP and a normal FBG level.

Ferroptosis inhibition reverses the effect of AGEs and serum from patients with OP and T2DM on hFOB1.19 osteoblasts. To determine the role of ferroptosis in the impairment of hFOB1.19osteoblast biological activities induced by AGEs 
A

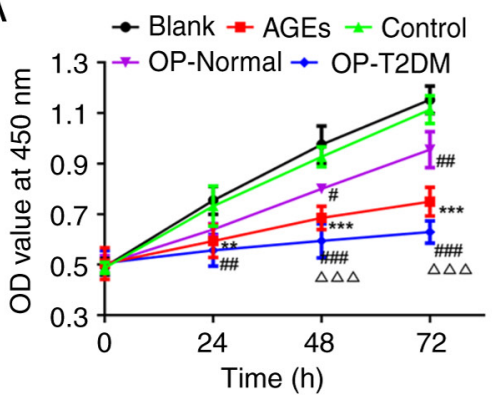

B

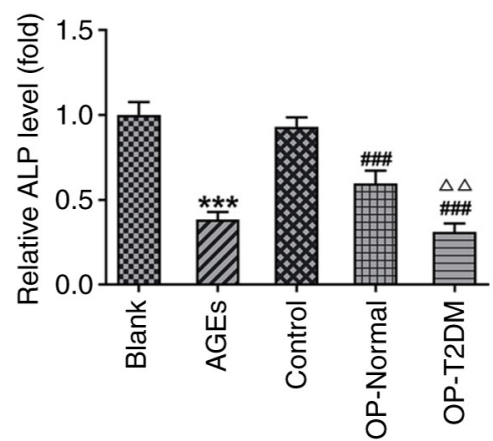

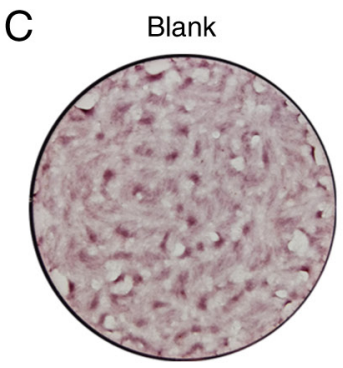
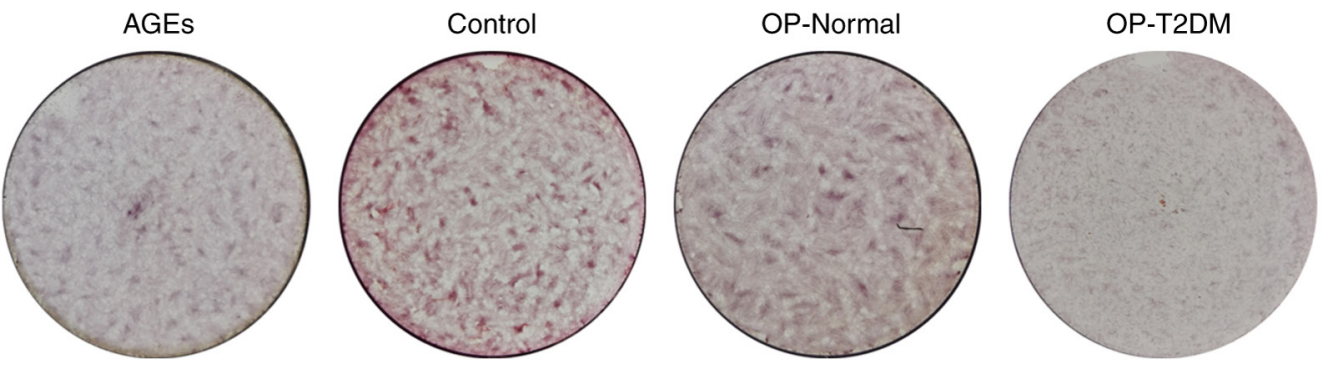

Figure 2. AGEs and serum from patients with OP inhibit the proliferation, differentiation and mineralization of hFOB1.19 osteoblasts. (A) A Cell Counting Kit-8 assay was used to detect cell proliferation. (B) The cellular ALP level of hFOB1.19 osteoblasts was measured using an ALP kit. (C) Alizarin red staining was performed to observe the formation of mineralized nodules. ${ }^{* *} \mathrm{P}<0.01$ and ${ }^{* * *} \mathrm{P}<0.001$ vs. Blank; ${ }^{*} \mathrm{P}<0.05,{ }^{\# \#} \mathrm{P}<0.01$ and ${ }^{\# \# \#} \mathrm{P}<0.001$ vs. Control; ${ }^{\Delta \Delta} \mathrm{P}<0.01$ and ${ }^{\Delta \Delta \Delta} \mathrm{P}<0.001$ vs. OP-Normal. AGEs, advanced glycation end products; OP, osteoporosis; ALP, alkaline phosphatase; OD, optical density; T2DM, type-2 diabetes mellitus.

and serum from patients with OP and T2DM, the ferroptosis inhibitor, DFO was added to the culture medium in the hFOB1.19 cells treated with AGEs and serum from patients with OP and T2DM (20). The CCK-8 and ALP assays, and Alizarin red staining results showed that the presence of DFO promoted cell proliferation, ALP production and mineralized nodule formation in the hFOB1.19 osteoblasts from the AGE and OP-T2DM groups (Fig. 4A-C). Cell apoptosis was measured using TUNEL staining, and the results showed that DFO decreased the number of apoptotic cells in the AGEs and OP-T2DM groups; however, the result was not significant ( $\mathrm{P}>0.05)$, (Fig. 4D). By contrast, DFO significantly reduced cellular iron, MDA and oxLDL levels, and increased GSH/GSSG levels in the hFOB1.19 osteoblasts from the AGE and OP-T2DM groups (Fig. 4E-H), indicating a DFO-induced inhibition of ferroptosis.

\section{Discussion}

OP is a common debilitating bone disease characterized by loss of bone mass and degradation of bone architecture. The involvement of AGEs in OP, particularly diabetes-related OP has been previously reported (21-23). In the present study, the inhibitory effect of AGEs on osteoblast proliferation, differentiation and mineralization was demonstrated, and the role of ferroptosis in these effects was identified. The results suggested that therapeutically targeting AGEs and AGE-induced ferroptosis in osteoblasts may promote bone formation and even relieve OP.

Patients with T2DM are more susceptible to OP, and the formation and accumulation of AGEs have been reported to cause diabetes-related OP (24). Increased AGE production is observed in diabetic rats, and AGE levels are negatively correlated with BMD (25). Higher levels of serum AGEs are found in patients with OP and osteopenia compared with those in healthy women (26). Consistently, the results in the present study showed increased serum AGE levels in patients with OP and a negative correlation with BMD. Furthermore, a higher concentration of FBG was observed in patients with OP compared with that in healthy individuals, and a positive correlation between AGEs and FBG was observed in patients with OP. The results further confirmed the crucial role of AGEs and hyperglycemia in OP.

OP is also a disease caused by an imbalance between osteoclasts and osteoblasts. Osteoblasts can contribute to bone formation by secreting various proteins that guide the deposition of bone extracellular matrix, such as ALP, osteocalcin and osteopontin (27). The results from the present study showed that AGEs and serum from patients with OP, which showed a higher concentration of serum AGEs compared with that in healthy individuals, significantly inhibited hFOB1.19 osteoblast proliferation, differentiation and mineralization. Furthermore, the effect of serum from patients with OP and T2DM was markedly stronger compared with serum from patients with OP and a normal FBG level, demonstrating the detrimental effect of hyperglycemia on human bone formation. These results were in accordance with those of previous studies, which showed that hyperglycemia and AGEs suppressed osteoblast differentiation and mineralization, accompanied by an enhanced RAGE expression (28-30). It has been reported that AGEs are diverse compounds that are generated via a non-enzymatic reaction between reducing sugars and the amine residues on proteins, lipids and nucleic acids (9). Intra- and extracellular AGEs accumulate in an age-dependent manner in human 

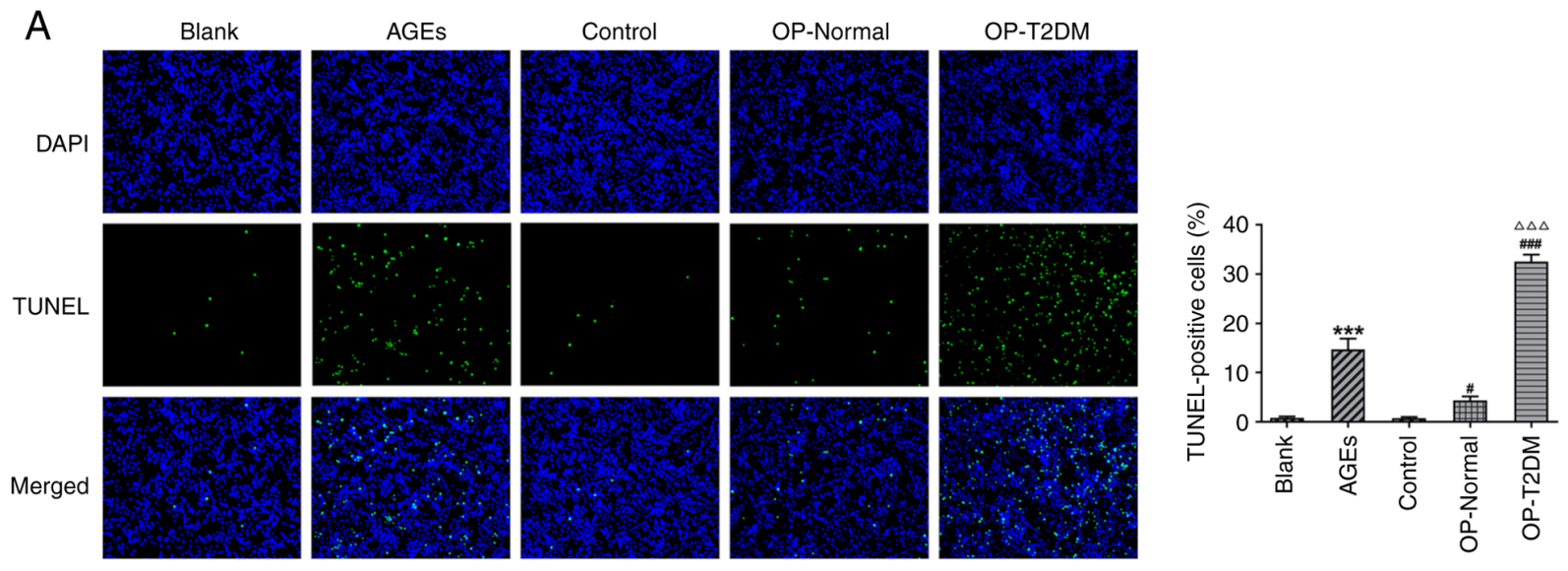

B
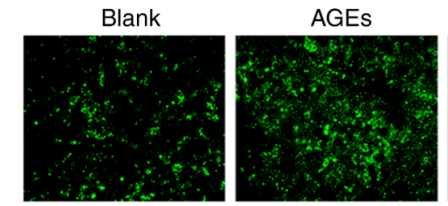

Control
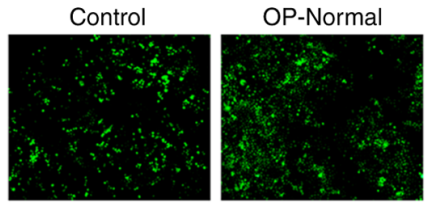

OP-T2DM
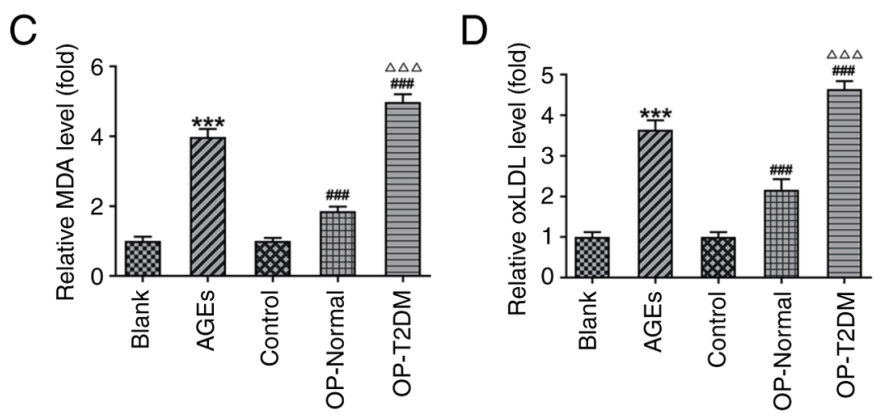

E
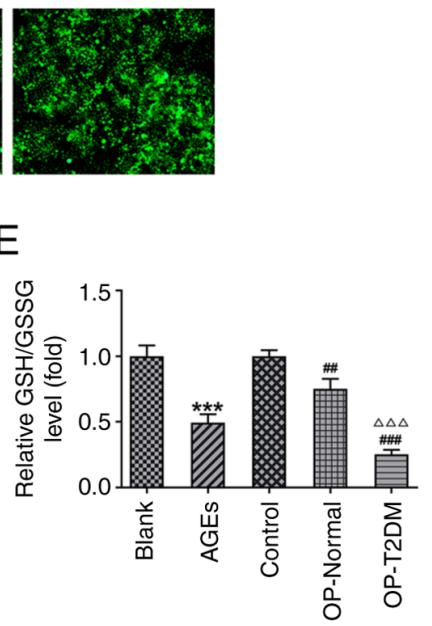

$\mathrm{F}$
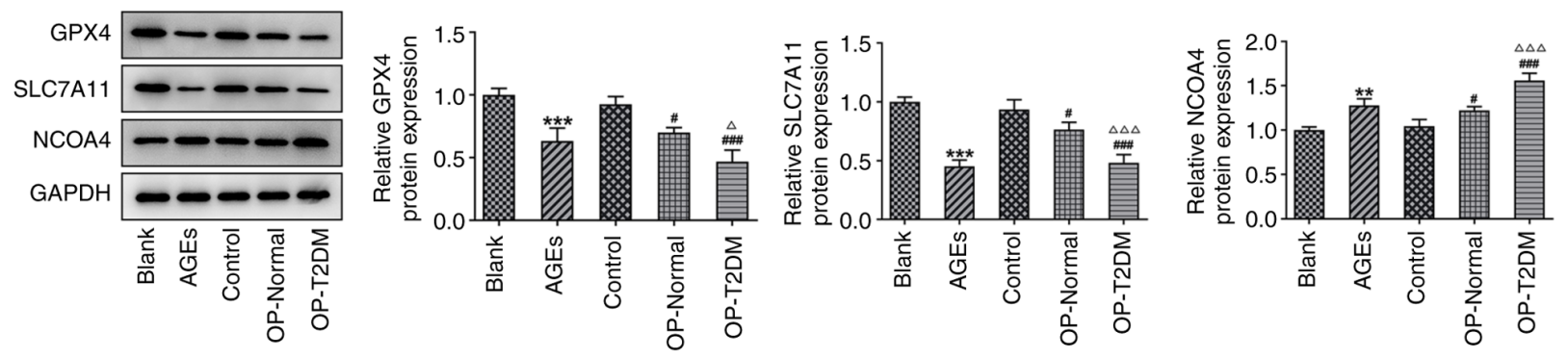

G
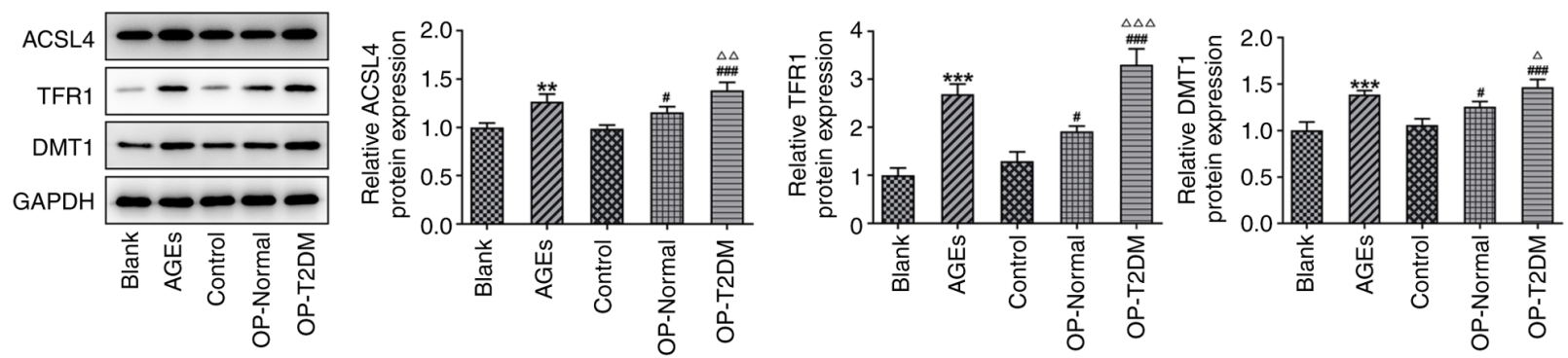

Figure 3. AGEs and serum from patients with OP induces apoptosis and ferroptosis in hFOB1.19 osteoblasts. (A) Representative images and quantitative analysis of TUNEL staining in hFOB1.19 cells from different treatment groups. (B) Cellular free iron levels in hFOB1.19 osteoblasts in different treatment groups. The relative levels of (C) cellular MDA, (D) ox-LDL and (E) GSH/GSSG in hFOB1.19 osteoblasts in different treatment groups. (F and G) The expression levels of proteins associated with ferroptosis was measured using western blot analysis. ${ }^{* *} \mathrm{P}<0.01$ and ${ }^{* * *} \mathrm{P}<0.001 \mathrm{vs}$. Blank; ${ }^{\#} \mathrm{P}<0.05$, ${ }^{\# \#} \mathrm{P}<0.01$ and

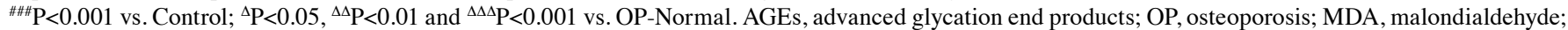
ox-LDL, oxidized low density lipoprotein; GSH/GSSG, glutathione/oxidized GSH; T2DM, type-2 diabetes mellitus; GPX4, GSH peroxidase; SLC7A11, solute carrier family 7 member 11; NCOA4, nuclear receptor coactivator 4; ACSL4, acyl-CoA synthetase long-chain family member 4; TFR1, transferrin receptor; DMT1, divalent metal transporter 1. 
A

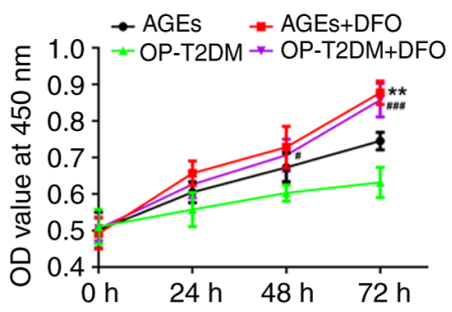

$\mathrm{B}$ 응

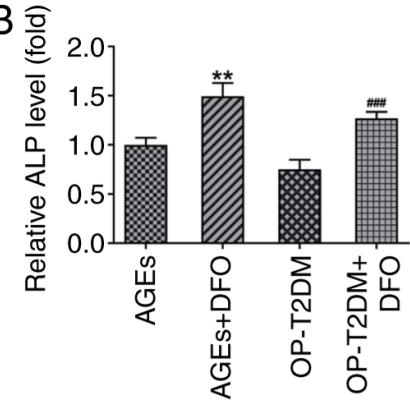

C
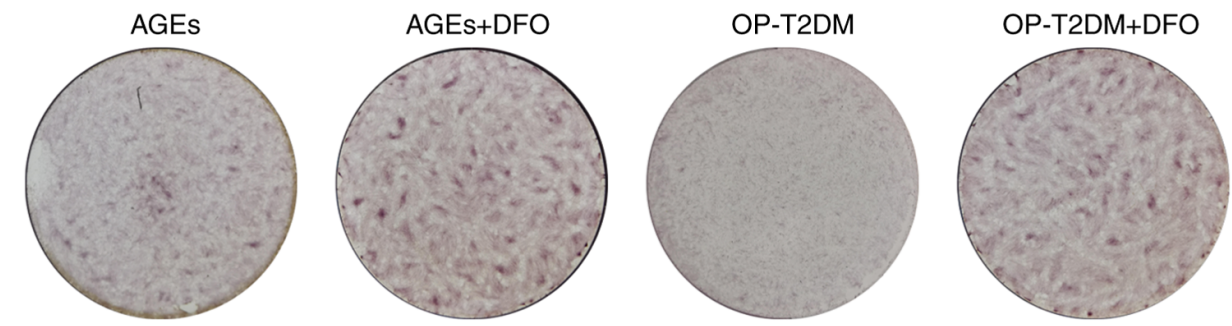

D

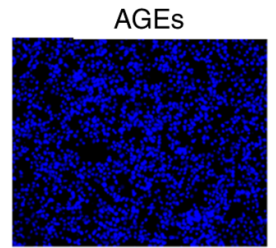

AGEs+DFO

OP-T2DM
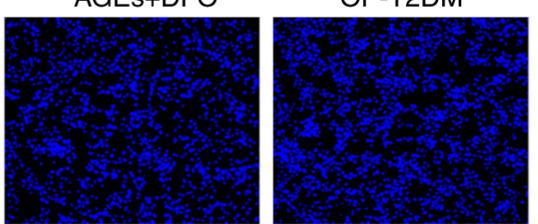

OP-T2DM+DFO
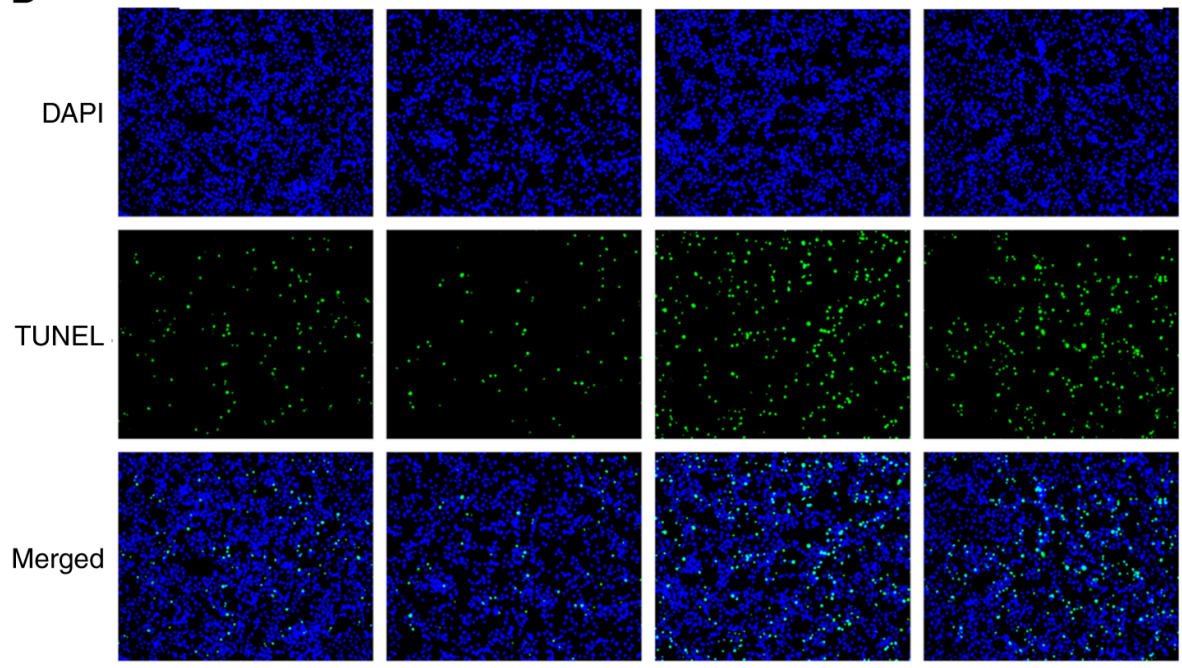

E

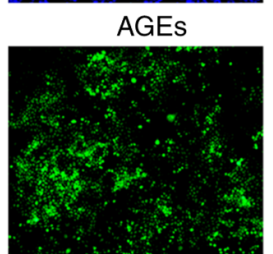

AGEs+DFO
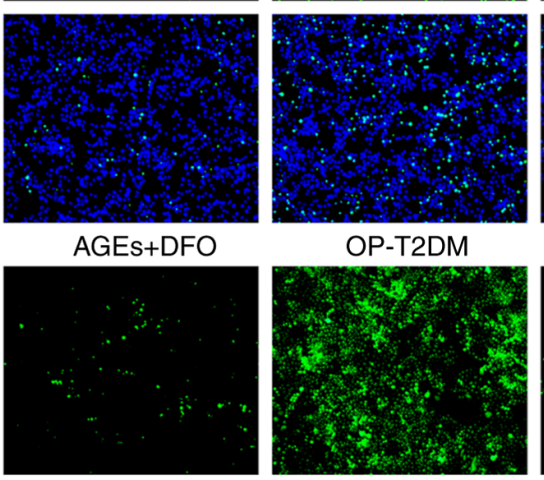

F

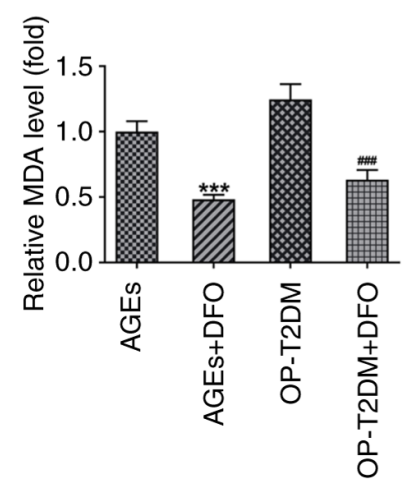

$G$ 음
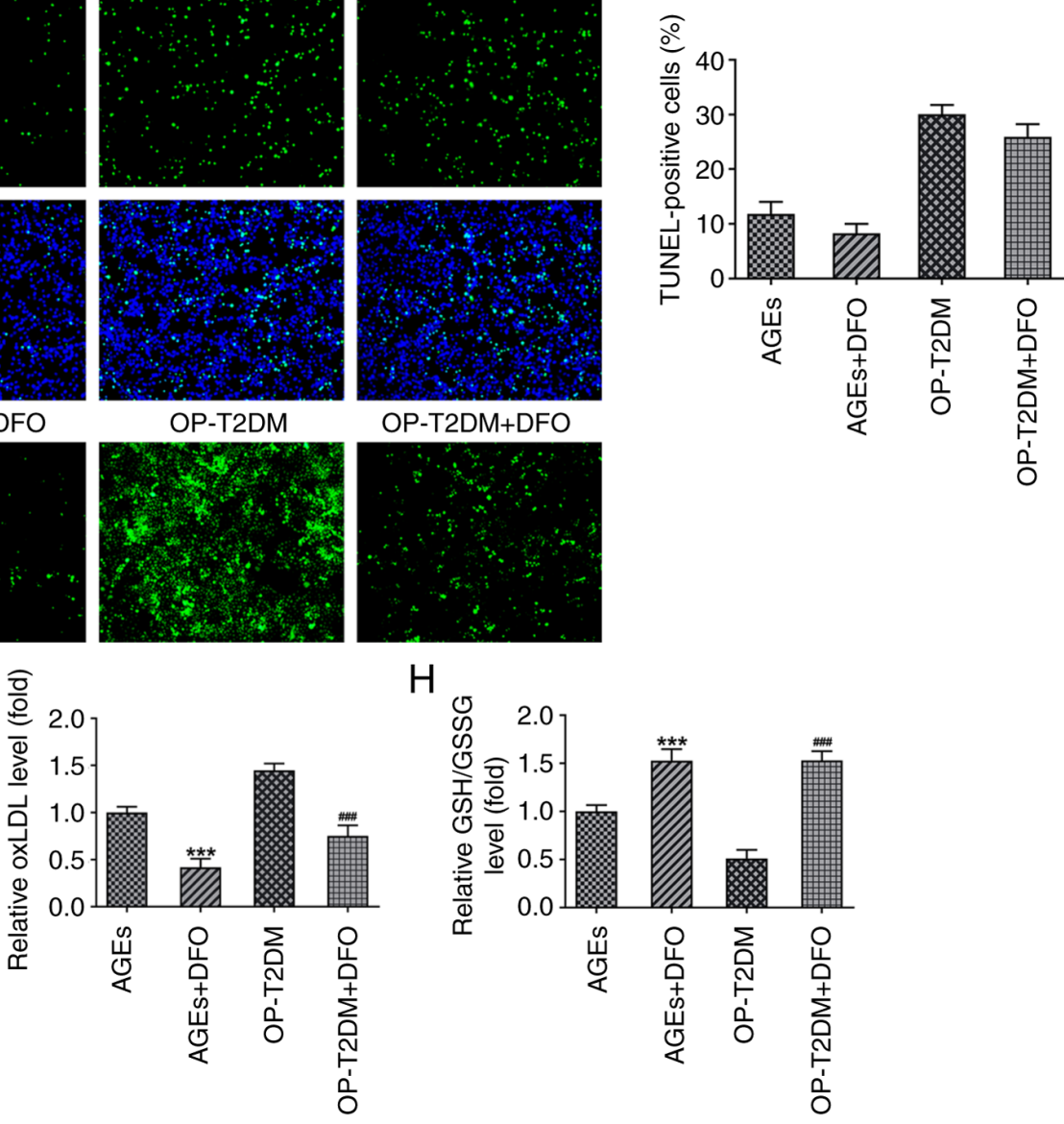

OP-T2DM+DFO

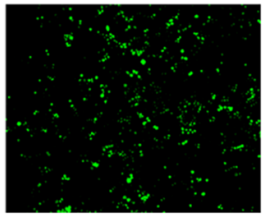

$\mathrm{H}$

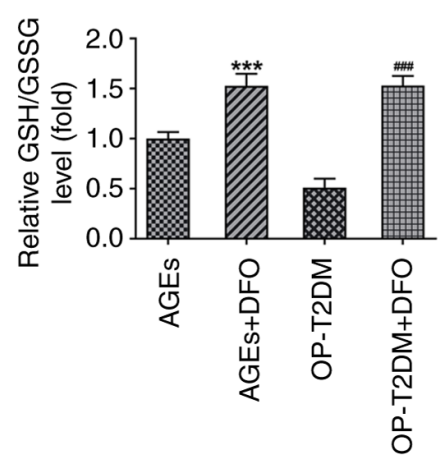

Figure 4. Ferroptosis inhibitor, DFO, reverses the effect of AGEs and serum from patients with OP and T2DM on hFOB1.19 osteoblasts. (A) A Cell Counting Kit-8 assay was used to detect proliferation in hFOB1.19 cells in different treatment groups. (B) Cellular ALP level of hFOB1.19 osteoblasts was measured using a ALP kit in different treatment groups. (C) Alizarin red staining was used to observe the formation of mineralized nodules in different treatment groups. (D) Representative TUNEL staining and quantitative analysis for hFOB1.19 osteoblasts in different treatment groups. (E) The cellular free iron level inhFOB1.19 osteoblasts in different treatment groups. The relative level of cellular (F) MDA, (G) ox-LDL and (H) GSH/GSSG in hFOB1.19 osteoblasts in different treatment groups. ${ }^{* *} \mathrm{P}<0.01$ and ${ }^{* * *} \mathrm{P}<0.001$ vs. AGEs; ${ }^{*} \mathrm{P}<0.05$ and ${ }^{\# \#} \mathrm{P}<0.001$ vs. OP-T2DM. DFO, deferoxamine; AGEs, advanced glycation end products; OP, osteoporosis; T2DM, type-2 diabetes mellitus; ALP, alkaline phosphatase; MDA, malondialdehyde; ox-LDL, oxidized low density lipoprotein; GSH/GSSG, glutathione/oxidized GSH; OD, optical density. 
tissue including cartilage, thus contributing to bone fragility in patients with diabetes (31). Therefore, the disruption of osteoblast function caused by serum from patients with OP and T2DM may also be attributed to the high production of AGEs.

To elucidate the mechanisms underlying the destructive effect of AGEs on bone function, ferroptosis was investigated, as it is a process that has been found to be involved in OP pathogenesis in recent years $(13,15,17,32)$. The results from the present study showed that AGEs and serum from patients with OP triggered apoptosis, and increased the cellular level of free iron, MDA and oxLDL levels, and reduced GSH/GSSG level in hFOB1.19 osteoblasts. Furthermore, AGEs and serum from patients with OP resulted in a decrease in the protein expression levels of GPX4 and SLC7A11, but an increase in the protein expression levels of NCOA4, ACSL4, TFR1 and DMT1. The accumulation of intracellular iron and lipid peroxidates, such as MDA, oxLDL and GSH, which are normally synthesized by ACSL4 and dissipated by the antioxidant enzyme GPX4, is a key feature of ferroptosis (33). In addition, the downregulation of SLC7A11 ultimately leads to a suppression of GPX4 activity and ferroptosis activation. NCOA4 is an autophagy cargo receptor, TFR1 is a transferrin receptor and DMT1 transports metal ions across membranes in mammals; all are necessary for iron release into the cytoplasm (34). The potential association between AGEs level and iron overload has been suggested in previous studies $(35,36)$. AGEs were also reported to enhance heme oxygenase-1 (HO-1) mRNA expression in endothelial cells (37) and inhibited Nrf2 activation in $\mathrm{H} 9 \mathrm{C} 2$ cells (38). Nrf2 is a transcription factor that regulates iron metabolism genes in response to oxidative stress, and increased HO-1 activity has been shown to increase cellular iron levels (39). The Nrf2/HO-1 signaling pathway has been reported to increase the cellular iron levels and promote ferroptosis $(13,40)$. The aforementioned studies have found an association between AGEs and ferroptosis; however, to the best of our knowledge, the present study was the first to demonstrate the effect of AGEs on ferroptosis. Subsequently, the ferroptosis inhibitor DFO was found to not only suppress ferroptosis, but also induce a recovery of hFOB1.19 osteoblast proliferation, differentiation and mineralization in the AGE, and OP-T2DM groups. However, DFO had no significant effect on apoptosis in the hFOB1.19 cells treated with AGE and serum from patients with OP and T2DM. This may be due to the specific inhibition of DFO on ferroptosis without affecting apoptosis. This was in line with our hypothesis, suggesting that AGEs may disrupt osteoblast function by inducing ferroptosis. Nevertheless, the present results remain to be validated using in vivo animal models, which will be performed in future research.

In conclusion, the results from the present study demonstrated the inhibitory effect of AGEs on bone formation in OP. AGEs significantly suppressed proliferation, differentiation and mineralization in hFOB1.19 osteoblasts. In addition, ferroptosis influenced the effects of AGEs. To the best of our knowledge, the present study was the first to uncover the mechanisms mediating the effect of AGEs on osteoblast function and demonstrated the role of osteoblast ferroptosis in bone formation. Therapies targeting AGEs and ferroptosis may be valuable in relieving OP.

\section{Acknowledgements}

Not applicable.

\section{Funding}

No funding was received.

\section{Availability of data and materials}

The datasets used and/or analyzed during the current study are available from the corresponding author upon reasonable request.

\section{Authors' contributions}

WLu, WG and JJ contributed to the conception or design of the study and performed the experiments. WW, JJ, JY, WLi and YC contributed to data analysis and interpretation. WLu, WG and JJ drafted the manuscript and revised it critically for important intellectual content. All authors read and approved the final manuscript. WLu, WG and JJ confirm the authenticity of all the raw data.

\section{Ethics approval and consent to participate}

All the experiments were approved by the Ethics Committee of the Nanjing Pukou District Central Hospital (Jiangsu, China). Written informed consent was obtained from all participants.

\section{Patient consent for publication}

Not applicable.

\section{Competing interests}

The authors declare that they have no competing interests.

\section{References}

1. Baccaro LF, Conde DM, Costa-Paiva L and Pinto-Neto AM: The epidemiology and management of postmenopausal osteoporosis: A viewpoint from Brazil. Clin Interv Aging 10: 583-591, 2015.

2. Armas LA and Recker RR: Pathophysiology of osteoporosis: New mechanistic insights. Endocrinol Metab Clin North Am 41: 475-486, 2012.

3. Reid IR: A broader strategy for osteoporosis interventions. Nat Rev Endocrinol 16: 333-339, 2020.

4. Ensrud KE, Ewing SK, Taylor BC, Fink HA, Stone KL, Cauley JA, Tracy JK, Hochberg MC, Rodondi N and Cawthon PM; Study of Osteoporotic Fractures Research Group: Frailty and risk of falls, fracture, and mortality in older women: The study of osteoporotic fractures. J Gerontol A Biol Sci Med Sci 62: 744-751, 2007.

5. Johnston CB and Dagar M: Osteoporosis in older adults. Med Clin North Am 104: 873-884, 2020.

6. Kurra S, Fink DA and Siris ES: Osteoporosis-associated fracture and diabetes. Endocrinol Metab Clin North Am 43: 233-243, 2014.

7. Nowotny K, Jung T, Höhn A, Weber D and Grune T: Advanced glycation end products and oxidative stress in type 2 diabetes mellitus. Biomolecules 5: 194-222, 2015.

8. Goh SY and Cooper ME: Clinical review: The role of advanced glycation end products in progression and complications of diabetes. J Clin Endocrinol Metab 93: 1143-1152, 2008.

9. Yamamoto M and Sugimoto T: Advanced glycation end products, diabetes, and bone strength. Curr Osteoporos Rep 14: 320-326, 2016. 
10. Alikhani M, Alikhani Z, Boyd C, MacLellan CM, Raptis M, Liu R, Pischon N, Trackman PC, Gerstenfeld L and Graves DT: Advanced glycation end products stimulate osteoblast apoptosis via the MAP kinase and cytosolic apoptotic pathways. Bone 40: 345-353, 2007.

11. Notsu M, Yamaguchi T, Okazaki K, Tanaka K, Ogawa N, Kanazawa I and Sugimoto T: Advanced glycation end product 3 (AGE3) suppresses the mineralization of mouse stromal ST2 cells and human mesenchymal stem cells by increasing TGF- $\beta$ expression and secretion. Endocrinology 155: 2402-2410, 2014.

12. Meng HZ, Zhang WL, Liu F and Yang MW: Advanced glycation end products affect osteoblast proliferation and function by modulating autophagy via the receptor of advanced glycation end Products/Raf Protein/Mitogen-activated protein Kinase/ExtracellularSignal-regulated Kinase Kinase/Extracellular Signal-regulated Kinase (RAGE/Raf/MEK/ERK) pathway. J Biol Chem 290: 28189-28199, 2015.

13. Ma H, Wang X, Zhang W, Li H, Zhao W, Sun J and Yang M: Melatonin suppresses ferroptosis induced by high glucose via activation of the Nrf2/HO-1 signaling pathway in type 2 diabetic osteoporosis. Oxid Med Cell Longev 2020: 9067610, 2020.

14. Zhou B, Liu J, Kang R, Klionsky DJ, Kroemer G and Tang D: Ferroptosis is a type of autophagy-dependent cell death. Semin Cancer Biol 66: 89-100, 2020.

15. Lu J, Yang J, Zheng Y, Chen X and Fang S: Extracellular vesicles from endothelial progenitor cells prevent steroid-induced osteoporosis by suppressing the ferroptotic pathway in mouse osteoblasts based on bioinformatics evidence. Sci Rep 9: 16130, 2019.

16. Xia D, Wu J, Xing M, Wang Y, Zhang H, Xia Y, Zhou P and $\mathrm{Xu}$ S: Iron overload threatens the growth of osteoblast cells via inhibiting the PI3K/AKT/FOXO3a/DUSP14 signaling pathway. J Cell Physiol: Jan 29, 2019 (Epub ahead of print) doi: $10.1002 /$ jcp. 28217.

17. Wang X, Ma H, Sun J, Zheng T, Zhao P, Li H and Yang M: Mitochondrial ferritin deficiency promotes osteoblastic ferroptosis via mitophagy in type 2 diabetic osteoporosis. Biol Trace Elem Res 200: 298-307, 2022.

18. Tsukushi S, Katsuzaki T, Aoyama I, Takayama F, Miyazaki T, Shimokata K and Niwa T: Increased erythrocyte 3-DG and AGEs in diabetic hemodialysis patients: Role of the polyol pathway. Kidney Int 55: 1970-1976, 1999.

19. Kim HJ and Kim SG: Alterations in cellular $\mathrm{Ca}(2+)$ and free iron pool by sulfur amino acid deprivation: The role of ferritin light chain down-regulation in prooxidant production. Biochem Pharmacol 63: 647-657, 2002.

20. Sun Y, Zheng Y, Wang C and Liu Y: Glutathione depletion induces ferroptosis, autophagy, and premature cell senescence in retinal pigment epithelial cells. Cell Death Dis 9: 753, 2018.

21. Cheng YZ, Yang SL, Wang JY, Ye M, Zhuo XY, Wang LT, Chen $\mathrm{H}$, Zhang $\mathrm{H}$ and Yang L: Irbesartan attenuates advanced glycation end products-mediated damage in diabetes-associated osteoporosis through the AGEs/RAGE pathway. Life Sci 205 184-192, 2018

22. Sanguineti R, Puddu A, Mach F, Montecucco F and Viviani GL: Advanced glycation end products play adverse proinflammatory activities in osteoporosis. Mediators Inflamm 2014: 975872 2014.

23. Li Y, Wang L, Zhang M, Huang K, Yao Z, Rao P, Cai X and Xiao J: Advanced glycation end products inhibit the osteogenic differentiation potential of adipose-derived stem cells by modulating Wnt/ $\beta$-catenin signalling pathway via DNA methylation. Cell Prolif 53: e12834, 2020.

24. de Paula FJ, Horowitz MC and Rosen CJ: Novel insights into the relationship between diabetes and osteoporosis. Diabetes Metab Res Rev 26: 622-630, 2010.
25. Saito M, Fujii K, Mori Y and Marumo K: Role of collagen enzymatic and glycation induced cross-links as a determinant of bone quality in spontaneously diabetic WBN/Kob rats. Osteoporos Int 17: 1514-1523, 2006.

26. Yang DH, Chiang TI, Chang IC, Lin FH, Wei CC and Cheng YW: Increased levels of circulating advanced glycation end-products in menopausal women with osteoporosis. Int J Med Sci 11: 453-460, 2014

27. Lee WC, Guntur AR, Long F and Rosen CJ: Energy metabolism of the osteoblast: Implications for osteoporosis. Endocr Rev 38 : 255-266, 2017.

28. Sanguineti R, Storace D, Monacelli F, Federici A and Odetti P: Pentosidine effects on human osteoblasts in vitro. Ann NY Acad Sci 1126: 166-172, 2008.

29. Franke S, Rüster C, Pester J, Hofmann G, Oelzner P and Wolf G: Advanced glycation end products affect growth and function of osteoblasts. Clin Exp Rheumatol 29: 650-660, 2011.

30. Lee EJ, Kang MK, Kim YH, Kim DY, Oh H, Kim SI, Oh SY, $\mathrm{Na} \mathrm{W}$ and Kang YH: Coumarin ameliorates impaired bone turnover by inhibiting the formation of advanced glycation end products in diabetic osteoblasts and osteoclasts. Biomolecules 10: 1052,2020

31. Dariya B and Nagaraju GP: Advanced glycation end products in diabetes, cancer and phytochemical therapy. Drug Discov Today 25: 1614-1623, 2020.

32. Ni S, Yuan Y, Qian Z, Zhong Z, Lv T, Kuang Y and Yu B: Hypoxia inhibits RANKL-induced ferritinophagy and protects osteoclasts from ferroptosis. Free Radic Biol Med 169: 271-282, 2021.

33. Casati L, Pagani F, Fibiani M,Lo Scalzo R and Sibilia V: Potential of delphinidin-3-rutinoside extracted from Solanum melongena $\mathrm{L}$. as promoter of osteoblastic MC3T3-E1 function and antagonist of oxidative damage. Eur J Nutr 58: 1019-1032, 2019.

34. Hirschhorn T and Stock well BR: The development of the concept of ferroptosis. Free Radic Biol Med 133: 130-143, 2019.

35. Mirlohi MS, Yaghooti H, Shirali S, Aminasnafi A and Olapour S: Increased levels of advanced glycation end products positively correlate with iron overload and oxidative stress markers in patients with $\beta$-thalassemia major. Ann Hematol 97: 679-684, 2018.

36. Chen SH, Yuan KC, Lee YC, Shih CK, Tseng SH, Tinkov AA, Skalny AV and Chang JS: Iron and advanced glycation end products: Emerging role of Iron in androgen deficiency in obesity. Antioxidants (Basel) 9: 261, 2020.

37. He M, Siow RC, Sugden D, Gao L, Cheng X and Mann GE: Induction of HO-1 and redox signaling in endothelial cells by advanced glycation end products: A role for Nrf2 in vascular protection in diabetes. Nutr Metab Cardiovasc Dis 21: 277-285, 2011.

38. Ko SY, Chang SS, Lin IH and Chen HI: Suppression of antioxidant Nrf-2 and downstream pathway in H9c2 cells by advanced glycation end products (AGEs) via ERK phosphorylation. Biochimie 118: 8-14, 2015.

39. Suttner DM and Dennery PA: Reversal of HO-1 related cytoprotection with increased expression is due to reactive iron. FASEB J 13: 1800-1809, 1999.

40. Xu T, Ding W, Ji X, Ao X, Liu Y, Yu W and Wang J: Molecular mechanisms of ferroptosis and its role in cancer therapy. J Cell Mol Med 23: 4900-4912, 2019.

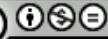

This work is licensed under a Creative Commons Attribution-NonCommercial-NoDerivatives 4.0 International (CC BY-NC-ND 4.0) License. 